\title{
Clinical profiling and outcomes of a fleeting diagnosis: Isolated right middle lobe pneumonia in the adult
}

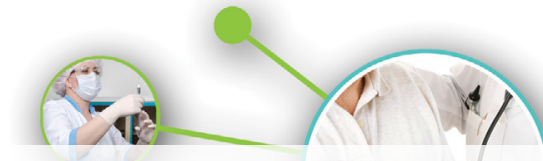

\begin{abstract}
Background: Little is known about clinical profiling and outcomes of adults with Isolated Right Middle Lobe Pneumonia (iRMLP). No previous comparison was made between hospitalized patients with iRMLP and other types of pneumonia.
\end{abstract}

Methods: We performed a retrospective analysis of patients with pneumonia during hospitalization. We checked the rate of iRMLP diagnosis by internal medicine physicians or a specialist in chest imaging. We performed a clinical profile of iRMLP patients, comparing their outcomes to other, non-iRMLP patients. We also checked the extent of diagnostic, interdiscipline, rate of diagnosis agreement.

Results: 286 pneumonia patients were included, of which 16 (5.5\%) were diagnosed as having iRMLP by a chest imaging specialist while 45 patients were diagnosed as having iRMLP by a specialist in internal medicine. The rate of agreement between assessments was fair (Cohen's kappa coefficient=0.41). Overall $20(7 \%)$ patients died during hospitalization. There was no correlation between the ascertained diagnosis of iRMLP and in-hospital mortality ( $p=0.3) .12$ patients (4.2\%) necessitated mechanical ventilation; 32 patients (11.2\%) were re-hospitalized within 14 days and 37 patients (13\%) died during the next 90 days. For 133 patients (46.5\%) the Length of Hospitalization (LOS) was over 5 days and for 59 patients (20.6\%) the LOS was longer than over 8 days. No outcome correlated with the ascertained diagnosis of iRMLP.

Conclusion: iRMLP in the adult is a fleeting diagnosis which should rely on expert opinion. Clinical profiling and outcomes do not differ significantly between iRMLP and other hospitalized patients with pneumonia.

Keywords: pneumonia, right-middle-lobe pneumonia, chest x-ray, mortality, cohen's kappa coefficient, internal medicine

\section{Introduction}

Establishing a diagnosis of pneumonia and characterizing its type (either lobar or atypical) and location (by lobe) are parts of the daily clinical practice in internal medicine departments. Frequently, imaging studies' results have a profound effect on the diagnosis, prognosis and treatment planning for pneumonia patients. Nevertheless, studies have shown that inter-observer agreement rates regarding specific pneumonia (and in fact, the mere existence of pneumonia) diagnosis is low [1-6].

One specific type of pneumonia, Isolated Right Middle Lobe Pneumonia (iRMLP), may be given special attention. iRMLP is associated with the Right Middle Lobe (RML) syndrome, a syndrome of RML atelectasis that might be related to the presence of space-occupying lesions and/or enlarged lymph nodes at the right lung hilum. Our future intentions were, in case iRMLP would be a common finding, to establish its prognosis and accordingly, recommend specific, further investigations for such patients, (e.g., computed tomography and/ or bronchoscopy). In light of the above, we sought to characterize the diagnostic accuracy (in terms of inter-observer agreement), clinical profiling and clinical outcomes for hospitalized, community-acquired, iRMLP patients.

\section{Methods}

We performed a retrospective analysis of a cohort of consecutive patients who were hospitalized to an internal medicine department of a large, tertiary hospital. All patients had a discharge diagnosis of "Pneumonia" (relevant ICD-9 codes: 486; 481; 484.8).
S Cohen ${ }^{1}$, S Lieberman ${ }^{2}$, A Brom ${ }^{3}$, A Biber $^{3}$, Y Sidi $^{4}$ and $G$ Segal ${ }^{3 *}$

${ }^{1}$ Sackler School, Tel-Aviv University, Israel

${ }^{2}$ Department of Radiology, Sheba Medical Center, Israel

${ }^{3}$ Department of Internal Medicine " $T$ ", Sheba Medical Center, Israel

${ }^{4}$ Management Wing, Sheba Medical Center, Israel

*Author for correspondence:

Gad.segal@sheba.health.gov.il 
The cohort size was determined by the following parameters: the estimated ratio between iRMLP and non-iRMLP would be $1: 4$, reflecting the number of pulmonary lobes. Also, we estimated that the expected mortality rate of hospitalized patients with communityacquired pneumonia would be $10 \%$. In order to maintain a significant level of $5 \%$ and power of $80 \%$, using Winpepi software, the size of our intended cohort should have been 355 patients. Since the actual ratio between iRMLP and noniRMLP pneumonia patients was different from the anticipated (the rate of iRMLP diagnosis, by either physician, was low), we analyzed an overall group of 286 patients.

The inclusion criteria of the study were: 1). Age over 18 years; 2). A discharge diagnosis of pneumonia (relevant ICD-9 codes: 486; 481; 484.8); 3). A chest x-ray was done during index hospitalization. All chest X-Rays were interpreted by a specialist in internal medicine. All chest X-Rays which did not show obvious pathology in lobes other than RML were also interpreted by a specialist in chest radiology. The Cohen's kappa coefficient was used to assess the inter-observer rate of agreement regarding the diagnosis of iRMLP. Continuous variables were assessed for normal distribution by a histogram and Q-Q diagrams and were described using either mean \pm Standard Deviation (SD) or median \pm Inter-Quartile Range (IQR). Categorical variables were described using frequency and percentages. Patients' clinical characteristics (recurrent hospitalizations or need of invasive ventilation) were compared using the $\mathrm{X}^{2}$ test or the Fisher's exact test for categorical parameters and using a T-test or Mann-whitney test for continuous variables. The association between iRMLP and mortality was assessed using a univariate Cox regression analysis. Variables that were found to have significant correlation with clinical outcomes $(\mathrm{p}<0.2)$ in the univariate analysis, along with parameters that are already known (in the literature) to correlate with clinical outcomes, were all included into multivariate analysis. The association between length of hospitalization and categorical variables was assessed using a Mannwhitney test or the Kruskal Wallis test while for continuous variables we used the Spearman coefficient. The length of hospitalization was divided by the median and a logistic regression was used to assess the correlation between prolonged hospitalization, iRMLP, and other study variables. Variables with statistical significance lower than 0.1 were included in the logistic regression. Analyses were performed with SPSS (IBM Corp. Released 2015. IBM SPSS Statistics for Windows, Version 23.0. Armonk, NY: IBM Corp.).

\section{Results}

A total of 286 pneumonia patients were included in the study. Patients' characteristics are presented in TABLE 1, according to their ascertained, imaging diagnosis. As detailed, the two groups did not differ, in a statistically significant manner, in their baseline demographics, smoking status, background diagnoses, in-hospital management (both diagnostic and therapeutic), laboratory tests and clinical outcomes. The vital signs also did not differ between groups aside from the measured, room-air saturation: The saturation values amongst patients with an ascertained iRMLP were significantly lower than measured for non-iRMLP patients ( $90 \%$ vs. $94 \%, \mathrm{p}=0.009)$. TABLE 2 detail risk factors potentially associated with increased in-hospital mortality amongst the whole study cohort. Variables that were found to have statistical significance were: residency in a long-term facility $(40 \%$ vs. $18 \%$; $\mathrm{p}=0.03)$, background of ischemic heart disease (50\% vs. $21.4 \% ; \mathrm{p}=0.01)$, dementia $(45 \%$ vs. $18 \% ; \mathrm{p}=0.007)$, chronic renal failure $(25 \% v s$. $9.1 \% ; \mathrm{p}=0.04$ ), older age (mean: 86.5 vs. 79; $\mathrm{p}=0.004)$, lower weight (Kg: 60 vs. 70; $\mathrm{p}=0.02)$, lower blood albumin (g/dL: 3.0 vs. 3.2; $\mathrm{p}=0.02$ ) and lower Norton score on admission (10 vs. 17; $\mathrm{p}<0.001)$.

An ascertained diagnosis of iRMLP did not have a significant impact on clinical outcomes: in-hospital and 90 days mortality, length of hospital stay, rate of hospital re-admission and need for mechanical ventilation were all practically unaffected by this diagnosis.

\section{Discussion}

Community Acquired Pneumonia (CAP) is a common, often severe and potentially lethal disease. Nevertheless, means for obvious diagnosis, radiographic evaluation and treatment are far from being definitive and consensual [6]. In their recent review, Wunderink et al. challenge the validity and accuracy of radiographic diagnosis of CAP, relying on two studies: one by Claessens et al. who found a significant discordance between clinical CAP diagnosis and CT findings, occurring in nearly $40 \%$ of cases [7]. Another study, by Wesley et al. in which using a CT as the gold standard, the 
Clinical profiling and outcomes of a fleeting diagnosis: Isolated right middle lobe pneumonia in the adult

TABLE 1. Patients' characteristics according to ascertained, imaging diagnosis.

Definitive iRMLP patients $(\mathrm{N}=16$ )

Non-iRMLP patients $(\mathbf{N}=\mathbf{2 7 0})$

$\mathbf{p}$

Patients' Demographics

Gender (Male) n; (\%)

Long term facility $\mathrm{n}_{\mathbf{i}}(\%)$

Admission Norton score ; mean (IQR)

Age ; mean \pm SD

Weight ; mean \pm SD

$8(50 \%)$

$5(31.3 \%)$

$17.5(11.25-19)$

$79 \pm 13$

$68.7 \pm 13.8$

Smoking Status n; (\%)

Past smoker

Current smoker

Background diagnoses n; (\%)

Diabetes mellitus

Chronic obstructive pulmonary disease

Ischemia heart disease

Dementia

Status post Stroke

Congestive heart failure

Malignancy (other than lung cancer)

Lung cancer

Chronic kidney disease

In-hospital Management

CT scan; $\mathbf{n}$ (\%)

Intra-venous Antibiotics; $\mathbf{n}(\%)$

Per-Os Antibiotics; $\mathbf{n}(\%)$

Systemic steroids; $\mathbf{n}$ (\%)

Catecholamine treatment; $\mathbf{n}$ (\%)

Laboratory Tests

WBC (K/microl); median (IQR)

CRP (mg/L); median (IQR)

Room air saturation (\%); median (IQR)

SBP (mmHg); median (IQR)

DBP (mmHg); median (IQR)

ALT (IU/L); median (IQR)

TEMP ( ${ }^{\circ}$ Celsius); median (IQR)

ALB (gr/dL); median (IQR)

Positive blood cultures; $n$ (\%)

Clinical Outcomes

In-hospital mortality; n (\%)

90-days mortality; $\mathbf{n}(\%)$

Necessitating invasive ventilation; $\boldsymbol{n}(\%)$

LOS; Length of stay (days) $>5$ days; $\mathbf{n}(\%)$

LOS; Length of stay (days) $>\mathbf{8}$ days; $\mathbf{n}(\%)$

Re-hospitalization within 14 days; $\mathbf{n}$ (\%)

\begin{tabular}{|c|}
\hline $8(50 \%)$ \\
\hline $5(31.3 \%)$ \\
\hline $17.5(11.25-19)$ \\
\hline $79 \pm 13$ \\
\hline $68.7 \pm 13.8$ \\
\hline
\end{tabular}

$3(18.8 \%)$

$1(6.3 \%)$

I

4

$5(31.3 \%)$

$2(12.5 \%)$

$4(25 \%)$

$2(12.5 \%)$

$3(18.8 \%)$

$2(12.5 \%)$

$3(18.8 \%)$

$1(6.3 \%)$

$1(6.3 \%)$

\begin{tabular}{|c|c|c} 
& $138(51.1 \%)$ & 0.93 \\
\hline & $51(18.9 \%)$ & 0.21 \\
\hline & $76(10-19)$ & 0.419 \\
\hline & $72.2 \pm 17.6$ & 0.37 \\
\hline
\end{tabular}

\begin{tabular}{c}
2 (12.5\%) \\
$14(87.5 \%)$ \\
$13(81.3 \%)$ \\
\hline $2(12.5 \%)$ \\
\hline
\end{tabular}

0

$21(7.7 \%)$

$23(8.5 \%)$

0.81

0.88

92 (34.1\%)

51 (18.9\%)

$63(23.3 \%)$

$55(20.4 \%)$

35 (13\%)

47 (17.4\%)

55 (20.4\%)

$14(5.2 \%)$

$28(10.4 \%)$

0.82

0.74

$>0.99$

0.74

0.45

$>0.45$

$>0.99$

0.58

$>0.99$

SD: Standard Deviation; WBC: White Blood Cells; CRP: C Reactive Protein; SBP: Systolic Blood Pressure; DBP: Diastolic Blood Pressure

TABLE 2. Patients' characteristics according to in-hospital mortality.

Patients' characteristics; $\mathbf{n}(\%)$

Male gender; $\mathbf{n}(\%)$

Origin from a long-term facility; $\mathbf{n}(\%)$

Smoking (ever); $\mathbf{n}$ (\%)

Smoking (current); $\mathbf{n}(\%)$

Diabetes mellitus; n (\%)

Chronic obstructive lung disease; $\mathbf{n}(\%)$

Ischemic heart disease; $\mathbf{n}(\%)$

Dementia; $\mathbf{n}(\%)$

State post stroke; $\mathbf{n}(\%)$

\begin{tabular}{|l|l|}
\hline dead \\
$7(35 \%)$ \\
\hline $8(40 \%)$ \\
\hline $2(10 \%)$ \\
\hline $0(0 \%)$ \\
$7(35 \%)$ \\
$4(20 \%)$ \\
\hline $10(50 \%)$ \\
\hline $9(45 \%)$ \\
$2(10 \%)$ \\
\hline
\end{tabular}

\begin{tabular}{c}
\hline $13.4(10.76-17.72)$ \\
\hline $136.2(95.5-218.8)$ \\
$90(88-92)$ \\
\hline $124.5(100.25-169)$ \\
\hline $68.5(59.25-86)$ \\
$17.5(13-29.5)$ \\
\hline $37.3(36.75-38.475)$ \\
\hline $3.35(2.75-3.675)$ \\
$1(6.3 \%)$
\end{tabular}

$12.95(8.80-17.53)$

$128.6(50.2-207.8)$

$127(112-144)$

$67(58-76)$

18 (12-30)

$3.2(3-3.6)$

\begin{tabular}{|c|c|c|}
\hline 0 & $20(7.4 \%)$ & 0.61 \\
\hline $2(12.5 \%)$ & $35(12.9 \%)$ & $>0.99$ \\
\hline 0 & $12(4.4 \%)$ & $>0.99$ \\
\hline $4(31 \%)$ & $128(47 \%)$ & $>0.99$ \\
\hline $1(6.2 \%)$ & $55(20.3 \%)$ & 0.75 \\
\hline
\end{tabular}




\begin{tabular}{|c|c|c|c|}
\hline Congestive heart failure; $\mathbf{n}(\%)$ & $6(30 \%)$ & $43(16.2 \%)$ & 0.12 \\
\hline Known Malignancy; n (\%) & $4(20 \%)$ & $54(20.3 \%)$ & $>0.99$ \\
\hline Chronic renal failure; $\mathbf{n}(\%)$ & $5(25 \%)$ & $24(9.1 \%)$ & 0.04 \\
\hline Positive blood culture; n (\%) & $3(15 \%)$ & $13(4.9 \%)$ & 0.09 \\
\hline P.O antibiotics; $\mathbf{n}(\%)$ & $8(40 \%)$ & $229(86.1 \%)$ & 0.001 \\
\hline Systemic steroids; $\mathbf{n}(\%)$ & $7(35 \%)$ & $69(25.9 \%)$ & 0.37 \\
\hline Known lung cancer; $\mathbf{n}(\%)$ & $4(6.8 \%)$ & $11(4.8 \%)$ & 0.52 \\
\hline Age (years); median (IQR) & $86.5(77.5-91.5)$ & $79(67.5-86)$ & 0.004 \\
\hline Weight (Kg); median (IQR) & $60(55.5-65)$ & $70(60-83)$ & 0.02 \\
\hline WBC (K/microl) ; median (IQR) & $11.35(7.48-16.435)$ & $13(8.89-17.67)$ & 0.27 \\
\hline CRP (mg/L) ; median (IQR) & $121.53(44.02-177.577)$ & $129.33(50.88-210.2)$ & 0.79 \\
\hline Albumin (g/dL) ; median (IQR) & $3(2.5-3.5)$ & $3.2(3-3.6)$ & 0.02 \\
\hline Admission Norton score ; mean (IQR) & $10(8-13)$ & $17(11-19)$ & 0.001 \\
\hline
\end{tabular}

sensitivity of the detection of opacity by chest radiography was only $43.5 \%$ [8].

Focusing on the issue of Inter-observer agreement rate, it tends to be low between different physicians from different disciplines, such as radiology residents and radiology experts: Novack et al. [1] made a prospective study of 262 patients clinically suspected of having pneumonia. They compared the X-ray diagnosis made for these patients by the admitting physician, a radiologist, a pulmonologist and an expert in infectious diseases. They found out that the agreement rates between different disciplines were very low (kappa values ranged from 0.09 to 0.44$)$. In another study, Ojutiku et al. [2] showed that a specialist panel agreement rate with a radiology resident was quite low (kappa=0.43) and was lowest in cases of segmental pneumonia. Moncada et al. [3] assessed the inter-observer agreement rate of a pulmonologist and radiologist interpretations of 211 radiographs of patients with Community Acquired Pneumonia. They found out that the agreement rate was generally poor and was better in cases of pleural effusion and infiltrates located in the right upper lobes and both lower lobes. Their findings are consistent with ours, regarding the low consistency and poor interobserver agreement rate we found for RML pneumonia. It should be noted that low interobserver agreement rates, regarding the X-ray diagnosis of pneumonia are also low in the pediatric population, as shown in the studies by Levinski et al. [4] and by Williams et al. [5].
In the case of iRMLP, reliability of chest radiography interpretation FIGURE $\mathbf{1}$ and their implications for therapy become even more problematic. Most relevant literature includes anecdotal case studies: Dell' Omo et al. [9] described a case of iRMLP diagnosed as acute exogenous lipoid pneumonia as a result of pyro fluid inhalation by a fire-eater. Kim et al. [10] described an iRML pneumonia that was diagnosed, using tomography, as arising from bronchial obstruction by a carcinoid tumor. Cunha et al. [11] described a case of a middleaged male that presented with a non-resolving, RML and right lower lobe pneumonia that was eventually diagnosed as suffering from bronchoalveolar carcinoma.

RML pneumonia could be a part of a rather rare syndrome in adults: the RML Syndrome. This syndrome includes imaging and clinical evidence of RML damage (collapse, bronchiectasis, and hemoptysis), often necessitating surgical intervention. Einarsson et al. [12] described recurrent infections of the RML as a major sign of the RML syndrome, as it seemed also in the case described earlier by Kim et al. [10]. Reddy et al. [13] described a case of recurrent iRML which was part of an RML syndrome, arising from a broncholith that was obstructing the main RML bronchus. Regarding patients included in this study, the possibility of RML syndrome was not evident and considered irrelevant in the vast majority of patients.

Regarding the significant risk of death 


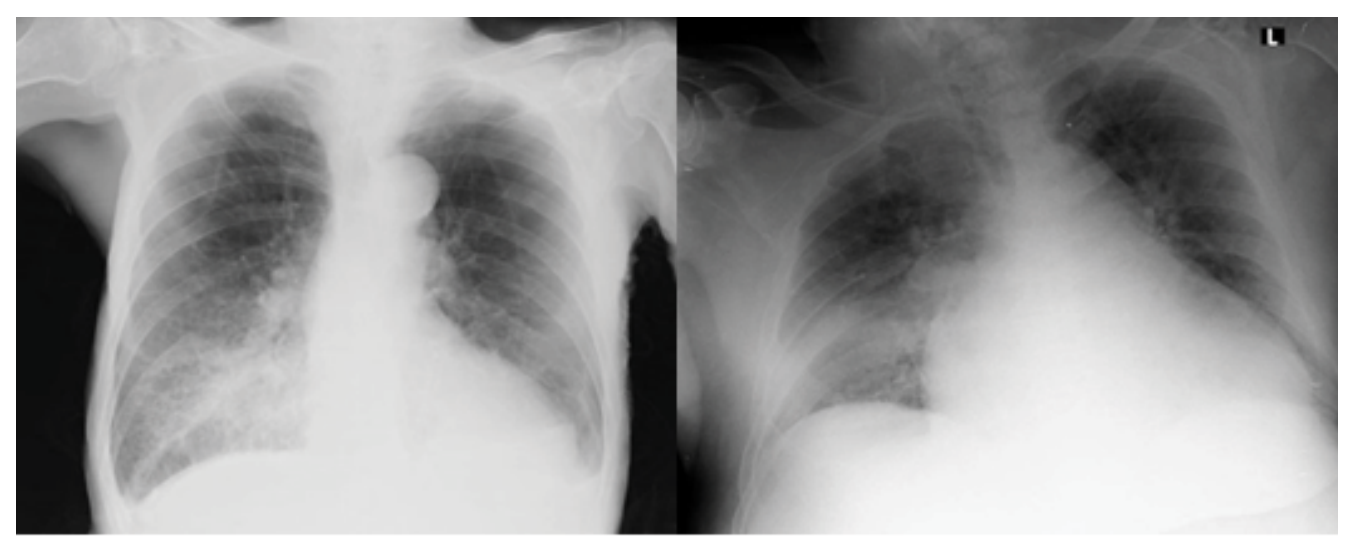

FIGURE 1. Two chest X-rays suggestive of iRML of our study cohort patients.

amongst patients with a background of ischemic heart disease: This issue was discussed by Wunderink et al. [6] who detailed the risk factors pneumonia patients to have for acute coronary syndrome. They even suggest prescribing antiplatelet therapies for such patients, although clinical studies were not undertaken for answering this question.

\section{Conclusion}

Isolated right middle lobe pneumonia is a diagnosis that should rely on expert interpretation of chest X-rays only. Nevertheless, the impact of accurate diagnosis, according to our study results, is low: variable clinical outcomes do not differ in a significant manner between inpatients with this diagnosis and other types of lobar pneumonia. Further studies should be done in order to improve the diagnostic accuracy of community-acquired pneumonia in general.

Risk factors for mortality amongst Community Acquired Pneumonia in our study cohort did not differ significantly from those published earlier. A higher rate of mortality amongst patients with a background diagnosis of ischemic heart disease should prompt future research and attention.

\section{Study Limitations}

This was a retrospective study and therefore, results are mainly relevant for clinical associations and causality could not be inferred. Also, the rate of iRMLP (5.5\%) was much lower than initially anticipated. We did not use advanced imaging (like CT scans) to ascertain the radiographic diagnosis. The cause for that is the fact that we wanted to do, as much as possible, a "real-life" study. There is no doubt that a future study, involving CT scans would potentially be more accurate in iRMLP diagnosis and therefore, clinical characterization of this clinical entity.

\section{Ethics, Consent and Permissions}

Prior to data collection and analysis, this study was approved by the Helsinki committee of the Chaim Sheba medical center. As a retrospective study, informed consent was not needed. This publication does not contain any personal/ individual data.

\section{Competing Interests}

None of the authors have any competing interests. 


\section{References}

Novack V, Avnon LS, Smolyakov A, et al. Disagreement in the interpretation of chest radiographs among specialists and clinical outcomes of patients hospitalized with suspected pneumonia. Eur J Intern Med. 17, 43-47 (2006)

Ojutiku O, Haramati LB, Rakoff S, et al. Radiology residents' on-call interpretation of chest radiographs for pneumonia. Acad Radiol. 12, 658-664 (2005).

Moncada DC, Rueda ZV, Macías A, et al. Reading and interpretation of chest X-ray in adults with community-acquired pneumonia. Braz J Infect Dis. 15, 540-546 (2011).

Levinsky Y, Mimouni FB, Fisher D, et al. Chest radiography of acute paediatric lower respiratory infections: experience versus interobserver variation. Acta Paediatr. 102, e310-314 (2013)

Williams GJ, Macaskill P, Kerr M, et al.
Variability and accuracy in interpretation of consolidation on chest radiography for diagnosing pneumonia in children under 5 years of age. Pediatr Pulmonol. 48, 1195-1200 (2013).

Wunderink RG, Waterer G. Advances in the causes and management of community acquired pneumonia in adults. BMJ. 358, j2471 (2017).

Claessens YE, Debray MP, Tubach F, et al. Early chest computed tomography scan to assist diagnosis and guide treatment decision for suspected community-acquired pneumonia. Am J Respir Crit Care Med. 192, 974-982 (2015).

Self WH1, Courtney DM, McNaughton $\mathrm{CD}$, et al. High discordance of chest $\mathrm{x}$-ray and computed tomography for detection of pulmonary opacities in ED patients: Implications for diagnosing pneumonia. $\mathrm{Am} \mathrm{J}$ Emerg Med. 31, 401-405 (2013).

Dell' Omo M, Murgia N, Chiodi M, et al. Acute pneumonia in a fire-eater. Int J Immunopathol Pharmacol. 23, 1289-1292 (2010).

Kim MP, Ernst A, DeCamp MM, et al. Endobronchial ultrasound-facilitated videoassisted lobectomy with wedge bronchoplasty for typical carcinoid tumor of the right middle lobe. Chest. 133, 1474-1476 (2008).

Cunha BA, Syed U, Mikail N. Bronchoalveolar carcinoma (adenocarcinoma) mimicking recurrent bacterial communityacquired pneumonia (CAP). Heart Lung. 41, 83-86 (2012).

Einarsson JT, Einarsson JG, Isaksson H, et al. Middle lobe syndrome: a nationwide study on clinic pathological features and surgical treatment. Clin Respir J. 3, 77-81 (2009).

Reddy AJ, Govert JA, Sporn TA, et al. Broncholith removal using cryotherapy during flexible bronchoscopy: A case report. Chest. 132, 1661-1663 (2007). 